\title{
CARDIAC AND NEUROENDOCRINE RESPONSES TO EXPOSURE THERAPY IN HEIGHT PHOBICS: DESYNCHRONY WITHIN THE 'PHYSIOLOGICAL RESPONSE SYSTEM'
}

\author{
James L. Abelson and George C. Curtis \\ Department of Psychiatry, Anxiety Disorders Clinic, University of Michigan, Ann Arbor, \\ MI 48109, U.S.A.
}

(Received 18 November 1988)

\begin{abstract}
Summary-Subjective, behavioral, cardiovascular and neuroendocrine responses were intensively monitored in two height phobics over a full course of exposure therapy and at follow-up. Both subjects showed rising cortisol responses and stable, non-extinguishing norepinephrine responses to height exposure over the course of treatment, while improvement occurred in subjective and behavioral response systems. They had differing heart rate responses. Despite desynchrony amongst anxiety response systems and within the physiological 'system' at treatment conclusion, both subjects had successful outcomes which were preserved at follow-up. Implications for the measurement of the 'physiological response system' in behavioral research are discussed.
\end{abstract}

\section{INTRODUCTION}

Anxiety researchers today usually measure anxiety in subjective, behavioral or physiological response 'systems' (Barlow, 1985). Measures within these systems often vary independently and may fail to improve in unison during the treatment of phobias-a phenomenon that has been called 'desynchrony' (Rachman and Hodgson, 1974; Hodgson and Rachman, 1974). Desynchrony may predict poor outcome or relapse following treatment of a phobia (Barlow, Mavissakalian and Schofield, 1980; Vermilyea, Boice and Barlow, 1984); and effective treatment may require specific techniques designed to treat each of the three anxiety response systems (Lang, 1968).

In behavioral research, the physiological response system has usually been measured using a single variable such as heart rate (Vallis and Segal, 1986) or galvanic skin response (Fowles, 1986). However, many other autonomic and neuroendocrine variables also respond to emotional stimuli (Levi, 1975) and fluctuate somewhat independently of each other (Nesse, Curtis, Thyer, McCann, Huber-Smith and Knopf, 1985). Ss with animal phobias respond to in vivo exposure with impressive subjective and behavioral manifestations of anxiety and increased secretion of growth hormone (Curtis, Nesse, Buxton and Lippman, 1979) and epinephrine and norepinephrine (Nesse et al., 1985). Cortisol and prolactin responses have been inconsistent (Curtis, Nesse, Buxton and Lippman, 1978; Nesse, Curtis, Brown and Rubin, 1980; Nesse et al., 1985) and thyroid stimulating hormone responses have not been seen (Nesse, Curtis and Brown, 1982). Acute stress generally does enhance secretion of cortisol, epinephrine, norepinephrine, and growth hormone (Curtis and Glitz, 1988) and increases cardiovascular reactivity. However, endocrine and cardiovascular responses during the psychologic stress of exposure therapy are not reliable and coordinated in a way that a simple theory of an anxiety physiological response system would predict (Nesse et al., 1985). Thus, desynchrony may apply to variables within the physiological 'response system' as well as between systems. Whether such within system desynchrony predicts outcome in the behavioral treatment of phobias has not been explored.

The present study utilized a single case design to assess subjective, behavioral, and physiological responses over the course of behavioral treatment and at follow-up. This design follows the recommendations of the 1981 NIMH Conference on Behavioral Approaches to Anxiety Disorders (Barlow and Wolfe, 1981). It extends previous observations by employing $S$ s with other than animal phobias, measuring both cardiovascular and neuroendocrine variables and making observations over the entire course of a treatment program and at follow-up. 


\section{METHOD}

The $S$ s were two men with height phobias. $S 1$, a 34 yr old social worker, had an 8 yr history of height phobia. His career advancement had been significantly hampered by his inability to go above the third floor level. $S 2$, a 19 yr old student, had a $9 \mathrm{yr}$ history of height phobia. He had become severely impaired by his fears when he left his rural home to attend a large university. Both $S$ s met DSM-III criteria (APA, 1980) for simple phobias.

Treatment consisted of therapist guided live exposure sessions of 1-2 hr duration. Upon arrival for each session the $S$ was seated and a 19 gauge butterfly needle was placed in a forearm vein. A $10 \mathrm{cc}$ blood sample was drawn, a stopcock was attached and the line was flushed with sterile heparinized saline and left in place for subsequent sampling. The $S$ s then filled out phobia rating scales (Marks and Mathews, 1979) and six semantic differential scales on which they rated their attitudes towards high places. The latter provided a measure of two attitude variables-cognitive evaluations (good-bad, awful-nice, pleasant-unpleasant) and assessment of dangerousness (safe-dangerous, threatening-unthreatening, harmful-harmless). $S s$ then sat quietly for $10 \mathrm{~min}$, after which a second blood sample was drawn.

A behavioral achievement test (BAT) was then conducted on a six story parking structure. $S \mathrm{~s}$ were given $10 \mathrm{~min}$ in which to ascend the parking structure using the elevator to minimize exercise effects, stopping on each floor to walk to the rail and look down. They were instructed to ascend as rapidly as their fear would allow them. Heart rate (Applied Systems Laboratories Series 4600 Pulse Rate Monitor) and subjective anxiety in subjective units of distress or SUDs (Wolpe, 1973) were recorded at 11 stations: At the entrance to the first floor elevator, as the $S$ stepped off the elevator on each of five floors, and as he looked down over the rail on each floor. The time needed to reach each floor and rail was also recorded. Behavioral achievement was scored by giving the $S 1$ point for each station reached plus $1 / 2$ point for each minute under the $10 \mathrm{~min}$ time limit required to reach his final station. Since a person with no fear of heights could complete the test in $4 \mathrm{~min}$, the maximum score attainable was 14 points. Immediately following the BAT in some sessions, $S 1$ was seated and a blood sample was drawn as described above. For practical reasons a post BAT sample could not be obtained for all sessions on both $S$ s.

A therapy session was then conducted using the six story parking structure and other appropriate height stimuli. Heart rate and SUDs were recorded at $5 \mathrm{~min}$ intervals throughout each treatment session. Near the midpoint and again just before completion of each therapy session the $S$ was seated and blood samples were drawn. At the end of the session $S$ and therapist completed phobia rating scales and the $S$ s completed attitude scales.

Several days after the final treatment a control session was conducted. Data were collected as in treatment sessions but with no exposure to phobic stimuli. The session was conducted entirely on ground level but otherwise followed exactly the protocol of earlier sessions, taking special care to precisely reproduce the amount of walking done during both the BAT and treatment sessions.

In order to control for circadian variation of hormone levels, all sessions were held at the same time of day for each $S(4: 30-7: 00$ p.m. for $S 1 ; 11: 30$ a.m. $-2: 00$ p.m. for $S 2)$.

Blood samples were drawn into chilled tubes containing glutathione (for epinephrine and norepinephrine assays) or heparin (for cortisol assay). They were centrifuged immediately after being drawn, except for post-BAT samples which were kept on ice until completion of the treatment sessions. Plasma was removed and frozen. Plasma cortisol levels were determined by competitive protein binding (Murphy, 1967), plasma growth hormone by radioimmunoassay (Schalch and Pardner, 1964), and plasma epinephrine and norepinephrine by a modification of the single isotope derivative assay of Passon and Peuler (1973).

\section{RESULTS}

Treatment was effective for both $S$ s. After $17 \mathrm{hr}$ of therapist-assisted live exposure $S 1$ was completely comfortable alone on the top floor of a 19 story building. After $12 \mathrm{hr}$ of treatment $S$ 2 could comfortably ascend to the top floor of the tallest building then be available for treatment (13th). General measures of change (Phobia rating scales, Fear Survey Schedule and SCL-90) showed substantial improvement for both $S$ s. Improvement was maintained at 6 and 8 months 


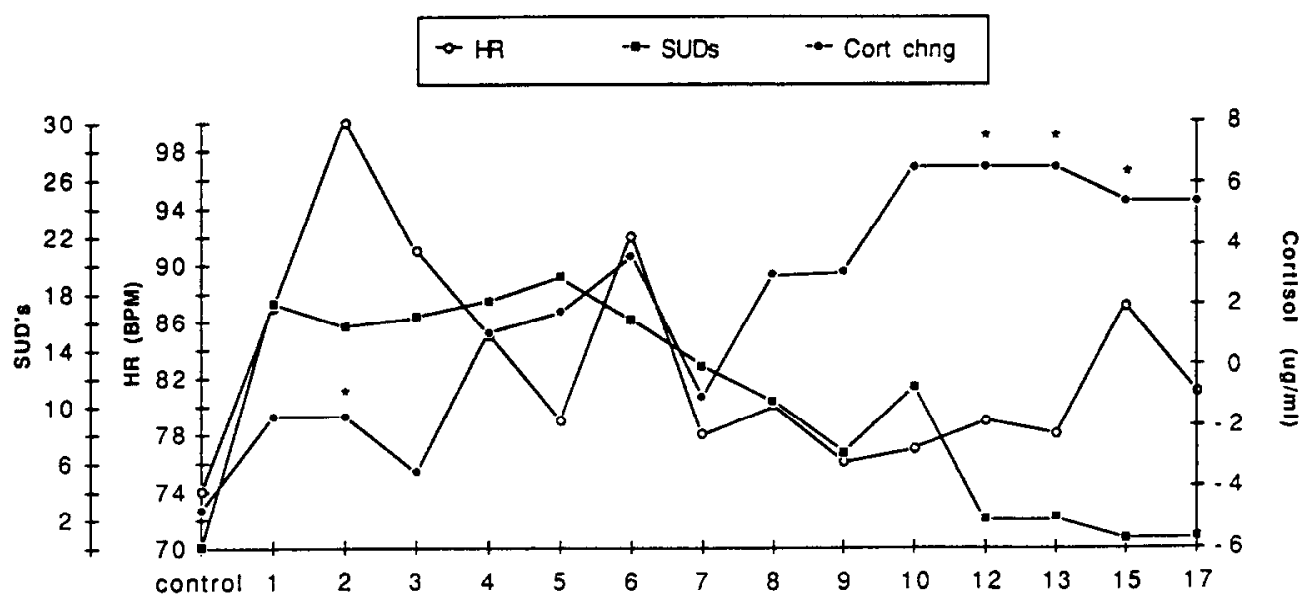

Fig. I. $S$ l's treatment session values for subjective anxiety (mean SUD's), heart rate (BPM), and cortisol (change from pre-session to session in $\mu \mathrm{g} \mathrm{ml}$ ). (Asterisks indicate missing data points with prior or subsequent session's value inserted.)

follow-up. These results are quite typical of daily clinical experience with this form of treatment and details are omitted to conserve space.

\section{Subject 1}

Mean SUDs during the initial BAT was 54. Subjective anxiety fell rapidly and this $S$ could complete the BAT with no subjective distress (mean SUDs of 0 ) by session 9 . He did not behaviorally master the BAT (achieve the maximal behavioral achievement score of 14) until session 15. At 8 month follow-up he could still reach the top station on the BAT but was slightly slower (behavioral achievement score of 12) and experienced mild anxiety (mean SUDs of 4.1 ).

Mean HR during the BAT was higher in all exposure sessions than in the non-exposure, equivalent exercise control session. The control value was 78 BPM. Exposure values were 81,90 , $87,91,85,90,82,87,87,84,83,82$ and $82 \mathrm{BPM}$ for sessions 1 through $10,12,13$ and 17 , respectively. At follow-up, mean HR during the BAT was lower than during any previous test (75 BPM).

Hormone responses to the BAT were assessed by subtracting the mean of the two pre-session blood levels from the post-BAT level and comparing this value to the equivalent value from the control session. This $S$ did not show a cortisol response to BAT exposure (control value $=1.3 \mu \mathrm{g} / \mathrm{ml} ;$ BAT values $=2.49,0.4$ and $3.57 \mu \mathrm{g} / \mathrm{ml}$ for sessions 1,10 and follow-up, respectively). He did show consistent norepinephrine and epinephrine responses which did not decline over the course of treatment. The NE BAT exposure values were $153,89,223$ and $123 \mathrm{pg} / \mathrm{ml}$ (for sessions 1, 10, 17 and follow-up, respectively) compared to an exercise equivalent control session value of $-6 \mathrm{pg} / \mathrm{ml}$. The $E$ exposure values were $30,60,43$ and $40 \mathrm{pg} / \mathrm{ml}$ compared to a control value of $2 \mathrm{pg} / \mathrm{ml}$.

$S$ l's treatment session data are reported in Fig. 1 (SUDs, HR and cortisol) and Table 1 (NE and E). Subjective anxiety fell fairly steadily over the course of treatment. Heart rate was higher in all exposure sessions than in the control session. It decreased over the first half of treatment but

Table 1. Norepinephrine and epinephrine, before treatment sessions, during treatment sessions, and change, over the course of treatment for $S 1$

\begin{tabular}{cccccccc}
\hline & \multicolumn{3}{c}{ NE $(\mathrm{pg} \mathrm{ml})$} & & \multicolumn{3}{c}{ E (pg ml) } \\
\cline { 2 - 4 } \cline { 7 - 8 } Session & Pre & During & Change & & Pre & During & Change \\
\hline Control & 315 & 292 & -23 & & 30 & 50 & 20 \\
1 & 270 & 424 & 154 & & 46 & 98 & 52 \\
3 & 373 & 669 & 296 & & 53 & 76 & 23 \\
5 & 247 & 318 & 71 & & 57 & 36 & -21 \\
8 & 202 & 273 & 71 & & 56 & 76 & 20 \\
9 & 209 & 352 & 143 & & 50 & 114 & 64 \\
10 & 344 & 598 & 254 & & 59 & 84 & 25 \\
17 & 370 & 501 & 131 & & 41 & 52 & 11 \\
\hline
\end{tabular}


then increased somewhat over the final 7 sessions. The HR peak in session 6 corresponded to first exposure to the roof of the parking structure and the peak in session 15 corresponded to first exposure to a new and substantially taller height stimulus.

Cortisol response to exposure in treatment sessions was assessed by subtracting the mean of the two pre-session blood levels from the mean of the mid-session and post-session levels. The expected diurnal variation of cortisol would predict a decline from pre-session to session values, and this decline was seen in the control session (reflected in the negative change score). Treatment generally reversed this expected decline and did so more strongly in later sessions. Although pre-session cortisol blood levels themselves declined from initially high values, this falling baseline cannot explain the increasing response to exposure because the baseline was stable (at $911 \mu \mathrm{g} / \mathrm{dl}$ ) during sessions 7-10 when the response was increasing most sharply.

$S 1$ showed a clear NE response to exposure during treatment sessions, without a detectable decline in this response over the course of treatment. A similar increase in NE was not seen in the non-exposure control session which reproduced the exercise demands of treatment sessions (Table 1). He did not show an E response to treatment sessions (Table 1). No growth hormone responses to the BAT or treatment sessions were detected.

In order to quantify relationships between the physiological response system and the behavioral and subjective systems, Pearson product-moment correlation coefficients were calculated. Cortisol responses to exposure in treatment sessions were significantly negatively correlated with the $S$ 's phobia scale ratings of avoidance $(r=-0.70, P<0.05)$ and impairment $(r=-0.72, P<0.05)$, and with the therapist's ratings of avoidance $(r=-0.74, P<0.05)$ and impairment $(r=-0.75$, $P<0.05$ ). The cortisol response to height exposure during treatment was also negatively correlated with the $S$ 's pre-session rating of the dangerousness of heights $(r=-0.83, P<0.01)$, i.e. the less dangerous he perceived heights to be before a session, the greater his cortisol response within that session. There were no other significant correlations between hormone variables and subjective or behavioral measures. Mean heart rate during treatment sessions was significantly, negatively correlated with behavioral achievement on the BAT $(r=-0.56, P<0.05)$, i.e. the more he could achieve on a pre-session behavioral test, the lower his HR during treatment session exposure. There were no other significant correlations between mean heart rates during the BAT or treatment sessions and any subjective or behavioral measures. Pre-session phobia scale ratings of avoidance and impairment by both the $S$ and therapist strongly predicted behavioral achievement on the BAT ( $r$ ranged from 0.83 to 0.93 with $P<0.001$ in each case).

\section{Subject 2}

This $S$ showed sensitization on the BAT. He pushed himself to the top station on the initial test, with mild subjective anxiety (mean SUDs $=18$ ) until he reached the top (SUDs there $=65$ ); but he then had a severe anxiety attack and fled to lower levels. He was unable to again reach the top station until three sessions later. He never achieved complete behavioral mastery of the BAT (he continued to approach the rail slowly, with a maximum score of 13 reached in his final session), but his subjective anxiety fell essentially to zero (mean SUDs $=0.1$ ). At 6 month follow-up he could complete the BAT a little faster (achievement score $=13.5$ ) with little anxiety (mean SUDs $=0.5$ ).

No BAT hormone data are available for $S 2$. This $S$ 's mean HR was consistently lower during the BAT (ranging from 69 to $75 \mathrm{BPM}$ ) than during the control session sham BAT (80 BPM). The only exception was session 3 (83 BPM). There was no directional trend in BAT HR over the course of treatment. The HR was lowest during the follow-up BAT (68 BPM).

$S 2$ 's treatment session data are reported in Fig. 2 (SUDs, HR and cortisol) and Table 2 (NE and E). Subjective anxiety fell fairly steadily over the course of treatment. Mean HR was lower than the control rate in all sessions except one, with no discernible trend over the course of treatment.

Normal diurnal variation of cortisol predicted higher values for $S 2$ (collected earlier in the day) than $S \mathrm{l}$; but this prediction was not borne out. Diurnal variation also predicted lower session than pre-session values and this was seen (reflected in negative change scores); but surprisingly, this expected fall disappeared over the course of treatment. The possibility that this $S$ inhibited cortisol secretion in response to exposure, and that this inhibition disappeared with treatment, is supported by the fact that all treatment session cortisol values were lower than the control session value. 


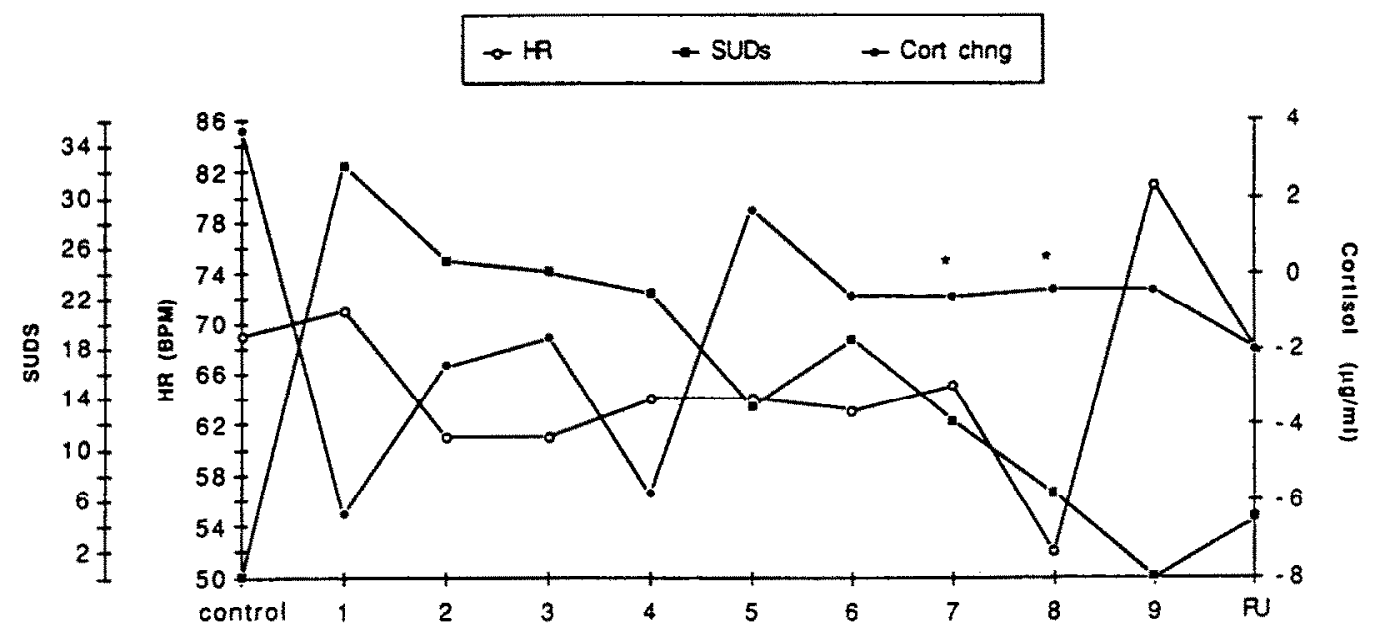

Fig. 2. $S$ 2's treatment session values for subjective anxiety (mean SUD's), heart rate (BPM), and cortisol (change from pre-session to session in $\mu \mathrm{g} / \mathrm{ml}$ ). (Asterisks indicate missing data points with prior or subsequent session's value inserted.)

$S 2$ showed a NE response to exposure during treatment sessions, over and above the exercise effects seen in the control session, with a hint of rising trend over the course of treatment in both pre-session and session values. He did not show an E response to treatment sessions (Table 2). No growth hormone response to treatment was detected.

Pearson product-moment correlation coefficients were calculated as for $S 1$. Cortisol response to exposure was not significantly correlated with any subjective or behavioral variable except for a negative correlation with the $S$ 's pre-session cognitive evaluation of heights $(r=-0.82$, $P<0.05$ ). As his attitude towards heights became more positive, less cortisol inhibition during treatment sessions was observed. The correlations between cortisol response and mean SUDs during the BAT and mean SUDs during treatment were also negative and approached significance ( $r=-0.73, P<0.07$ and $r=-0.68, P<0.10$, respectively). Cortisol level during treatment was significantly negatively correlated with post-session ratings of the dangerousness of heights $(r=-0.79, P<0.05)$ and with mean SUDs during sessions $(r=-0.76, P<0.05)$. NE level during treatment was significantly negatively correlated with pre-session cognitive evaluation of heights $(r=-0.95, P<0.05)$ and with mean SUDs during sessions $(r=-0.74, P<0.05)$. There were no other significant correlations between hormone variables and subjective or behavioral variables. Mean HR during treatment sessions was significantly negatively correlated with the $S$ 's pre-session phobia scale ratings of avoidance $(r=-0.78, P<0.05)$ and impairment $(r=-0.81, P<0.01)$. Other correlations between heart rates and subjective or behavioral measures were small and non-significant.

\section{DISCUSSION}

These results support the idea that the 'physiological response system' is not a single, synchronized system but has multiple components which may change in different directions as change occurs in the subjective and behavioral systems. Furthermore, they suggest that synchrony among all three response systems is not necessary for successful treatment of a phobia or for preservation of gains at follow-up.

Table 2. Norepinephrine and epinephrine, before treatment sessions, during treatment sessions, and change, over the course of treatment for $S 2$

\begin{tabular}{cccccccc}
\hline & \multicolumn{3}{c}{ NE $(\mathrm{pg} / \mathrm{m})$} & & \multicolumn{3}{c}{$E(\mathrm{pg} / \mathrm{ml})$} \\
\cline { 2 - 3 } \cline { 6 - 8 } Session & Pre & During & Change & Pre & During & Change \\
\hline control & 375 & 472 & 97 & & 56 & 88 & 32 \\
1 & 261 & 338 & 77 & & 48 & 54 & 6 \\
2 & 292 & 439 & 147 & & 42 & 72 & 30 \\
4 & 161 & 394 & 233 & & 14 & 73 & 59 \\
5 & 307 & 440 & 133 & & 22 & 41 & 19 \\
9 & 391 & 566 & 175 & & 89 & 73 & -16 \\
\hline
\end{tabular}


Desynchrony was striking for $S 1$. As his phobic avoidance and impairment declined, subjective anxiety declined, behavioral achievement increased, cortisol secretion during treatment sessions increased, heart rate response to exposure in treatment decreased but did not extinguish, and NE response to exposure in treatment remained present and unchanged. All three physiological measures responded to exposure to the feared stimulus, but they all responded differently as subjective and behavioral manifestations of anxiety were extinguished over the course of therapy. Furthermore, none of the three physiological variables were fully extinguished at the conclusion of a successful treatment. Such desynchrony may indicate a high risk of relapse or the need for further treatment (Barlow et al., 1980; Grey, Rachman and Sartory, 1981; Barlow, 1985). This $S$, however, reported no avoidance and no impairment at 8 month follow-up and was still able to complete the BAT with minimal difficulty.

This $S$ 's heart rate declined as behavioral achievement increased, but he showed an increase in HR when exposed to more challenging stimuli in sessions 6 and 15. There was also a trend towards increasing HR during the final treatment sessions when he was being exposed to the greatest heights. This end-of-treatment increase in HR corresponded to the slow withdrawal of the therapist's supportive presence during the closing stages of therapy. There is evidence that interpersonal contact can have a significant calming effect on cardiac function (for citations see Lynch, 1977). The withdrawal of the therapist's support may also have increased the performance demand characteristics of the situation, which can also increase HR (Borkovec, Stone, O'Brien and Kaloupek, 1974). During the 8 month follow-up BAT, however, HR was lower than during any previous BAT, despite high performance demand. It may be that with continued exposure in everyday life, this $S$ 's HR response finally extinguished. It is also possible that the interpersonal contact provided at follow-up played a role in the lowered HR. Desychrony was still seen at follow-up in that $\mathrm{E}$ and NE responses to the BAT remained unchanged. These findings support Vallis and Segal's (1986) belief that there are problems with the use of HR as the primary measure of the physiological response system (see Öhman, 1987 for a theoretical analysis of this problem). However, by demonstrating substantial desynchrony using neuroendocrine measures, our findings contradict their hypothesis that the high degree of desynchrony reported in previous research resulted from the reliance on HR to monitor physiological responses.

Although the results for $S 2$ were not as strikingly desynchronous as those for $S 1$, his data support the above conclusions. As his attitude towards height stimuli improved, his subjective anxiety during exposure decreased, his tendency to inhibit cortisol during exposure disappeared, and treatment session levels of both cortisol and NE increased. An increasingly positive attitude towards heights and lower subjective anxiety both predicted higher levels of NE during exposure sessions. Like $S 1$ his NE response to exposure did not extinguish over the course of treatment. Unlike $S 1$, exposure to heights failed to consistently raise HR above control levels. In fact, HR during exposure was almost always lower than control values. The striking individual differences between these two height phobics in HR responses to identical stimuli further demonstrates the difficulty of relying on $\mathrm{HR}$ to assess physiological anxiety. For both $S$ s two variables which we would expect to be correlated with each other and with other measures of anxiety, HR and NE, did not change in unison or in synchrony with subjective and behavioral measures when followed over a full course of exposure therapy. Cortisol, a classic stress hormone, increased as treatment success was achieved and subjective distress disappeared. Despite this desynchrony, both $S$ s overcame their phobia and were still doing well 6 and 8 months later.

As has been seen previously (Nesse et al., 1985), exposing phobic patients to their feared stimulus does produce physiological responses; but these responses are not reliable and consistent, may vary between individuals, and do not change in a simple and predictable way during the extinction of subjective and behavioral fear responses. Given the intensity of the subjective and behavioral responses seen in exposure therapy, the lack of more striking and consistent physiological responses is surprising. One explanation may involve differences between acute and chronic stressors. Repeated exposure to the acute release of stress hormones will lead to alterations in neurotransmitter receptor numbers and sensitivity. There is evidence that in anxiety patients repeated acute arousal may lead to chronic downregulation of receptors in the adrenergic and hypothalamicpituitary-adrenal systems (Curtis and Glitz, 1988). It is possible that an intensive exposure therapy that alters subjective and behavioral fear responses will cause regulatory changes in receptor 
function. The effects of such physiologic regulatory responses, as they interact with acute responsivity, on multiply determined outcome measures such as heart rate or even serum hormone levels is not readily predictable. In our $S$ s there was some evidence of increasing physiological responsivity at the end of treatment. It is possible that this reflects a return of normal responsivity in previously downregulated physiological systems, occurring as chronic, excessive subjective and behavioral fear responses are extinguished.

These ideas are speculative, but our limited data do suggest that the concept of three anxiety response systems as currently employed in the behavioral literature requires broadening. The physiological response system is not a unitary system but a complex web of systems. The same may well be true of the subjective and behavioral response 'systems' (Öhman, 1987). These 'systems' appear to interact in complex and as yet ill-defined ways that can be better delineated through additional extended and intensive studies of individual $S \mathrm{~s}$, with multiple measures taken over the full course of treatment and at follow-up.

Acknowledgement The author is indebted to Randolph Nesse, M. D. for invaluable assistance in the preparation of this manuscript for publication.

\section{REFERENCES}

APA (1980) Diagnostic and Statistical Manual of Mental Disorders (3rd edn). APA, Washington D.C.

Barlow D. H. (1985) The dimensions of anxiety disorders. In Anxiety and Anxiety Disorders (Edited by Tuma A. H. and Maser J. D.). Erlbaum, Hillsdale, N.J.

Barlow D. H. and Wolfe B. E. (1981) Behavioral approaches to anxiety disorders: a report on the NIMH-SUNY, Albany, Research Conference. J. consult. clin. Psychol. 49, 448-454.

Barlow D. H., Mavissakalian M. R. and Schofield L. (1980) Patterns of desynchrony in agoraphobia: a preliminary report. Behav. Res. Ther. 18, $44 !-448$.

Borkovec T., Stone N., O'Brien G. and Kaloupek D. (1974) Evaluation of a clinically relevant target behavior for analog outcome research. Behav. Ther. 5, 503-513.

Curtis G. C. and Glizz D. A. (1988) Neuroendocrine findings in anxiety disorders. Endocr. metab. Clin. N. Am. 17, 131-147.

Curtis G. C., Nesse R. M., Buxion M. and Lippman D. (1978) Anxiety and plasma cortisol at the crest of the circadian cycle: reappraisal of a classical hypothesis. Psychosom. Med. 40, 368-378.

Curtis G. C., Nesse R. M., Buxton M. and Lippman D. (1979) Plasma growth hormone: effects of anxiety during flooding-in-viro. Am. J. Psychiat. 136, 410-414.

Fowles D. C. (1986) The psychophysiology of anxiety and hedonic affect: motivational specificity. In Proc. Int. Symp. on Anxiety Disorders: Theory, Diagnosis and Treatment (Edited by Shaw B. F., Segal Z. V., Vallis T. M. and Cashman F. E.). Plenum Press, New York.

Grey S. J., Rachman S. and Sartory G. (1981) Return of fear: the role of inhibition. Behav. Res. Ther. 19, 135-144.

Hodgson R. and Rachman S. (1974) II: Desynchrony in measures of fear. Behav. Res. Ther. 12, 319-326.

Lang P. J. (1968) Fear reduction and fear behavior: Problems in treating a construct. In Research in Psychotherapy (Edited by Shlien J. M.), Vol. 3. American Psychological Association, Washington D.C.

Levi L. (1975) Emotions-Their Parameters and Measurement. Raven Press, New York

Lynch J. L. (1977) The Broken Heart: The Medical Consequences of Loneliness. Basic Books, New York.

Marks I. M. and Mathews A. M. (1979) A brief standard self-rating scale for phobia patients. Behav. Res. Ther. 17, 263-267.

Murphy B. E. P. (1967) Some studies of the protein-binding of steroids and their application to the routine micro and ultramicro measurement of various steroids in body fluids by competitive protein-binding radioassay. J. clin. Endocr. Metab. 27, 293-390.

Nesse R. M., Curtis G. C. and Brown G. M. (1982) Phobic anxiety does not effect plasma levels of thyroid stimulating hormone in man. Psychoneuroendocrinology 7, 69-74.

Nesse R. M., Curtis G. C., Brown G. M. and Rubin T. R. (1980) Anxiety induced by flooding therapy for phobias does not elicit prolactin secretory response. Psychosom. Med. 42, 25-31.

Nesse R. M., Curtis G. C., Thyer B. A., McCann D. S., Huber-Smith M. J. and Knopf R. F. (1985) Endocrine and cardiovascular responses during phobic anxiety. Psychosom. Med. 47, 320-332.

Ohman A. (1987) The psychophysiology of emotion: an evolutionary-cognitive perspective. Adv. Psychophysiol. 2, $79-127$.

Passon P. and Peuler J. (1973) A simplified radiometric assay for plasma norepinephrine and epinephrine. Annals Biochem. $51,618-620$.

Rachman S. and Hodgson R. (1974) I: synchrony and desynchrony in fear and avoidance. Behav. Res. Ther. 12, 311-318.

Schalch D. S. and Pardner M. L. (1964) A sensitive double antibody immunoassay for human growth hormone in plasma. Nature 203, 1141-1142.

Vallis T. M. and Segal Z. V. (1986) Anxiety disorders: future directions and closing comments. In Proc. Int. Symp. on Anxiety Disorders: Theory, Diagnosis and Treatment (Edited by Shaw B. F., Segal Z. V., Vallis T. M. and Cashman F. E.). Plenum Press, New York.

Vermilyea J. A., Boice R. and Barlow D. H. (1984) Rachman and Hodgson (1974) a decade later: how do desynchronous response systems relate to the treatment of agoraphobia? Behav. Res. Ther. 22, 615-621.

Wolpe J. (1973) The Practice of Behatior Therapy (2nd edn). Pergamon Press, New York. 